\title{
Research of Adaptive Gradient Projection Algorithm on Remote sensing Image Reconstruction
}

\author{
Yan Haixia1, a Liu Yanjun2, b \\ 1JiLin University Electronic Department, Changchun 130012,China \\ ${ }^{2}$ Changchun Institute of Optics, Fine Mechanics and Physics, the Chinese Academy of Sciences, \\ Changchun 130033, China; \\ ayanhx@jlu.edu.cn, bliuyanjun@ciomp.ac.cn
}

Keywords: remote sensing image, signal processing, gradient projection, compressed sensing

\begin{abstract}
In order to improve the low efficient and the noise effect of remote sensing image reconstruction, an algorithm of adaptive dual gradient projection for sparse reconstruction of compressed sensing theory is proposed. Point to the high frequency noise of remote sensing image, the ADGPSR algorithm pursuits the projection direction in two conjudate directions, thus the high frequency noise effect is overcame. Experiment results show that, compared with the GPSR algorithm, the ADGPSR algorithm on remote sensing image improves the signals reconstruction accuracy.
\end{abstract}

\section{Introduction}

Remote sensing is information science and technology which collects the target data by sensor, and obtains area information through the analysis of the data. There are high frequency noises in remote sensing image, the reason is that the noise and edge are the same appearance on gray space. In high resolution remote sensing image, the noises are distribution in high-frequency region. The image reconstruction is difficult, because the edge and noise are the same performance in image. When we overcome the noise effect, the image edge is effect at the same, thus the application of methods on remote sensing image have been subject to greater restrictions.

With the development of information technology, especially the development of digital signals process, how to complete the signals collection and signals digital procession is a important question. Nyquist theory is always the basis of sample theory, which works in the way of that in order to restore the originals signals, the rating speed will reach to the two speed of signals bandwidth[1 3]. But under the Nyquist theory, when the bandwidth of signal improves, the data information of signal became more and more, the information store and transmission is difficult to complete[4,5].

In recent years, the compressed sensing theory proposed by Candes, Romberg, et al (Compressed sensing) to overcome the shortcoming of Nyquist sampling theory, the basic idea of compressed sensing theory is that, sampling the signals with a lower sample rate, rather than the higher sample rate, then compressing method[6 8]. The basic of this theory is the sparse signals can be compressed. The theory can use far less than the Nyquist sampling frequency of data sampling, and still be able to accurately restore the signal[9 11]

Point to the efficient and the noise effect of remote sensing image reconstruction, we propose an algorithm of Adaptive Dual Gradient Projection for Sparse Reconstruction of compressed sensing theory. the ADGPSR algorithm pursuits the projection direction in two conjudate directions, thus the high frequency noise effect is overcame. Experiment results show that, compared with the GPSR algorithm, the ADGPSR algorithm on remote sensing image improves the signals reconstruction accuracy.

\section{Compressed sensing theory}

In compressed sensing theory, the signal must be sparse, or can be represented as sparse by some transforms. In general, the signals itself are not sparse, after a transformation (such as wavelet 
transform), the signals can be considered to be sparse, for example, after the wavelet transform, the transform results which contain $\mathrm{K}$ major results, and other $\mathrm{N}-\mathrm{K}$ results are set to zero. Assume that the original signal to be processed for $f \in R^{N}$, it's sparse basis is matrix $\Psi$, in this way the signal $f$ is sparse on the base $\Psi$, this process can be expressed as formula (1):

$$
f=\Psi x
$$

where $x$ is the decomposition of the system, it has a sparse features as formula (2):

$$
\|x\|_{0} \leq k
$$

The symbol $\|x\|_{0}$ is the norm of signal $l_{0}$, a number of non-zero value vector. After the signal is sparse representation, the random measurement can be complete by an observation matrix as formula (3):

$$
y=\Phi f=\Phi \Psi x=A x
$$

where $y$ is the measurement vector, $y \in R^{M}, M\langle\langle N$,because the signal has a sparse, the decoding process of the above-mentioned problems can be solved by the following formula:

$$
\min \|x\|_{0} \text { s.t. } y=A x
$$

When the coefficient vectors are get, the signal $f$ can be restored by $f=\Psi x$.

However, formula (4) is a NP-hard problem, it can't be solved within a limited time. One of the most important contribution of the compressed sensing theory is that the question of norm $l_{0}$ and be equal to question norm $l_{1}$, when the signals are sparse and the observation matrix satisfy certain conditions.

$$
\min \|x\|_{1} \text { s.t. } y=A x
$$

The symbol $\|x\|_{1}$ indicates that norm of $l_{1}$, the absolute value of the vector. Solving problems of the norm is a convex optimization problem, can be resolved by linear programming.

In the actual measurement process noise will be introduced inevitably, the model can be expressed as:

$$
y=O x+n
$$

Where $\mathrm{n}$ is the measurement noise vector. In this case, the signal reconstruction process can be expressed as:

$$
\min _{x}\|x\|_{1} \text { subject }\|y-A x\|_{2}^{2} \leq \varepsilon
$$

The algorithm, developed from the damped Newton method, For an unconstrained optimization of smooth nonlinear function $\mathrm{F}$, it is every step of the formula

$$
\delta^{(k)}=-H_{k}^{-1} F\left(z^{k}\right)
$$

Because the $H_{k}$ can not be calculated, need to figure out:

$$
\nabla F\left(z^{k}\right)-\nabla F\left(z^{k-1}\right) \approx \eta^{k}\left[z^{k}-z^{k-1}\right]
$$

Approximation algorithm of $H_{k}$ is not satisfied, increase of algorithm iterations.

$$
\begin{gathered}
\min c^{T} z+\frac{1}{2} z^{T} B z=F(z) \\
\text { where, } \quad z=\left[\begin{array}{l}
u \\
v
\end{array}\right], \quad b=A^{T} y, \quad c=\tau 1_{2 n}+\left[\begin{array}{l}
-b \\
b
\end{array}\right], \quad B=\left[\begin{array}{l}
A^{T} A,-A^{T} A \\
-A^{T} A, A^{T} A
\end{array}\right], \quad \mathrm{u} \text { and } \mathrm{v} \text {, respectively, }
\end{gathered}
$$
corresponding to the positive and negative part of the vector $\mathrm{x}, \mathrm{x}=\mathrm{u}-\mathrm{v}, \quad u \geq 0 v \geq 0,1_{2 n}{ }_{2 n-}$ dimensional unit column vector.

\section{Adaptive Dual Gradient Projection for Sparse Reconstruction}

The realizing process of ADGPSR as follows: 
Step 1: Choose parameters $\alpha_{\text {min }}, \alpha_{\max }, \alpha_{0} \in\left[\alpha_{\min }, \alpha_{\max }\right]$, set $k=0$.

$\alpha_{0} \quad$ Then, According to the formula (11) compute

$$
\alpha_{0}=\frac{\left(g^{(k)^{T}}\right) g^{(k)}}{\left(g^{(k)^{T}}\right) B g^{(k)}}
$$

Step 2: Compute step

$$
\delta^{(k)}=\left(z^{(k)}-\alpha^{(k)} \nabla f\left(z^{(k)}\right)-\alpha^{(k-1)} \nabla f\left(z^{(k-1)}\right)-z^{(k)}\right.
$$

Step 3:Search $\chi^{(k)} \in[0,1]$ in the interval to a minimum $\chi^{(k)}$, and set

$$
z^{(k+1)}=z^{(k)}+\lambda_{1}^{(k)} \delta^{(k)}+\lambda_{2}^{(k-1)} \delta^{(k-1)}
$$

Approximation $z^{(k+1)}$ solution then the algorithm terminates, $z^{(k+1)}$ is the reconstructed image, otherwise look for $\chi^{(k)}$.

$$
\alpha_{1}^{(k)}=\frac{\left\langle\nabla F\left(z^{(k)}\right)\right\rangle}{\left\|c^{(k)}\right\|_{2}^{2}} \alpha_{1}^{(k-1)}=\frac{\left\langle\nabla F\left(z^{(k-1)}\right)\right\rangle}{\left\|c^{(k-1)}\right\|_{2}^{2}}
$$

\section{Experiment and Analysis}

We know that the image is not sparse itself, in this article, we use the wavelet transform sparse the signal. And the random Gaussian matrix as the observation matrix, the variance distribution $(0,1 / N)$. The reconstruction algorithm uses the ADGPSR(Adaptive Gradient Projection for Sparse Reconstruction).

We use two remote sensing images to test the ADGPSR, the image size is $256 \times 256$, at the same time we use GPSR algorithm reconstruction image. The Fig.1 and Fig.2 shows the remote sensing image test results of ADGPSR . Fig1.(a) and Fig2.(a) are the original remote sensing images, Fig1.(b) and Fig2.(b) are the noise remote sensing images with noise variance equals to 2, Fig1.(c) and Fig2.(c) are the ADGPSR reconstruction results from noise remote sensing images, Fig1.(d) and Fig2.(d) are the GPSR reconstruction results from remote sensing image. With the same input remote sensing noise image which including roads and trees, the GPSR method PSNR is 16.52 and 21.26 differently, the GPSR method PSNR is 14.76 and 18.12 differently.

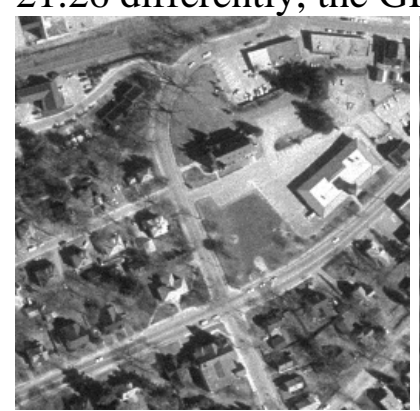

(a) Original image (PSNR=14.76)

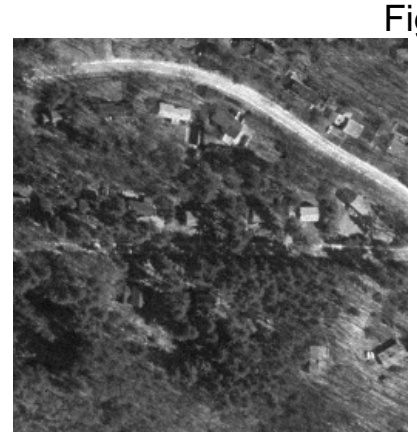

(a) Original image (PSNR=18.12)

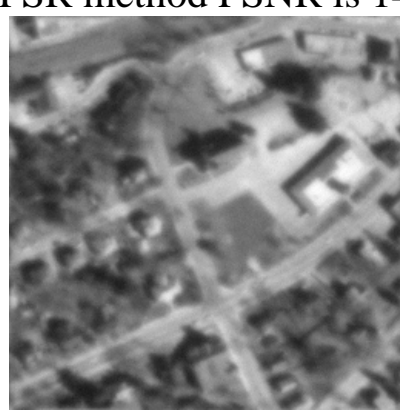

(b)noise image

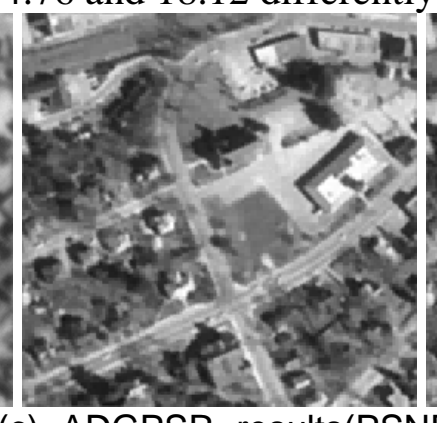

(c) ADGPSR results(PSNR=16.52

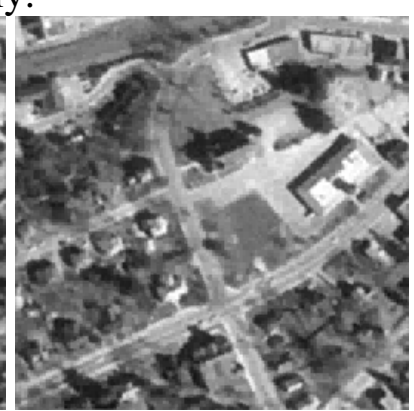

(d) GPSR results

Fig. 1 Road Remote Sensing image reconstruction results

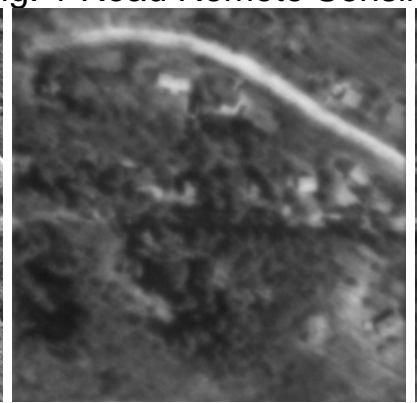

(b)noise image

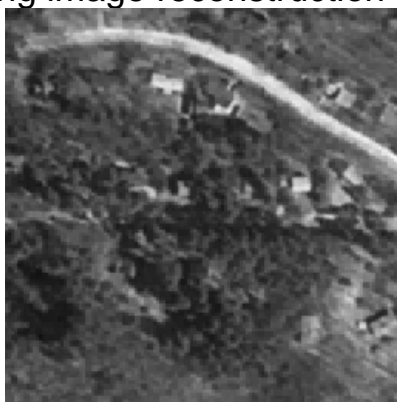

(c) ADGPSR results(PSNR=21.26)

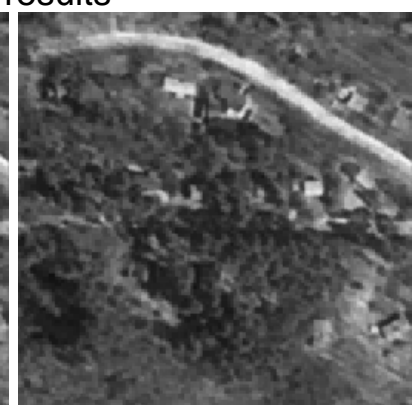

(d) GPSR results

Fig. 2 Tree Remote Sensing image reconstruction results 


\section{Summary}

In this paper, point to the low efficient and the noise effect of remote sensing image reconstruction, ADGPSR algorithm (adaptive dual gradient projection for sparse reconstruction ) is proposed, the ADGPSR algorithm pursuits the projection direction in two conjudate directions, thus the high frequency noise effect is overcame. The signals reconstruction quality of ADGPSR is higher than GPSR algorithm. We test the ADGPSR algorithm by different remote sensing images which including roads and trees, Experiment results shows that with the same input remote sensing noise image which including roads and trees, the GPSR method PSNR is 16.52 and 21.26 differently, the GPSR method PSNR is 14.76 and 18.12 differently.

Contact Author: Liu Yanjun, E-mail: liuyanjun@ ciomp.ac.cn

\section{References}

[1] D.Donoho,Compressed sensing[J].IEEE Trans.Inform.Theory.2006, 52 (4): 1289-1306.

[2] Candès,E.J.,Romberg,J.,Tao,T.,Signal recovery from incomplete and inaccurate measurements[J].Comm.Pure.Appl.Math.2005,59(8):1207-223.

[3] Donoho D,Huo X,Uncertainty Principles and Ideal Atomic Decompositions[J].IEEE Trans Inform Theory,2001,47(7):2845-2862.

[4] Lei, J. Generalized reconstruction algorithm for compressed sensing[J].Computers and Electrical Engineering, v 37, n 4, p 570-588, July 2011.

[5] M.Figueiredo,R.Nowak,S.Wright,Gradient projection for sparse reconstruction:Application to compressed sensing and other inverse problems[J],IEEE Journal of Slaected Topics in Signal Processing,2007. 12,1(4):586-597.

[6] Satyanarayana, J.V. Ramakrishnan, A.G. An efficient document image reconstruction and binarization method using Compressed Sensing[J].Proceedings of the IADIS International Conference Computer Graphics, Visualization, Computer Vision and Image Processing 2009, CGVCVIP 2009. Part of the IADIS MCCSIS 2009, p 287-291, 2009, Proceedings of the IADIS International Conference Computer Graphics, Visualization, Computer Vision and Image Processing 2009, CGVCVIP 2009. Part of the IADIS MCCSIS 2009.

[7] E.Candès,J.Romberg,T.Tao,Robust uncertainty principles:Exact signal reconstruction from highly incomplete frequency information[J],IEEE Trans.Inform.Theory,2006,52(2):489-509.

[8] Egiazarian, Karen. Compressed sensing image reconstruction via recursive spatially adaptive filtering[J].Proceedings - International Conference on Image Processing, ICIP, v 1, p I549-I552, 2006, 2007 IEEE International Conference on Image Processing, ICIP 2007 Proceedings.

[9] Ni Kangyu,Datta Somantika, Mahanti Prasun. etc.Using reed-muller sequences as deterministic compressed sensing matrices for image reconstruction[J].ICASSP, IEEE International Conference on Acoustics, Speech and Signal Processing - Proceedings, p 465-468, 2010, 2010 IEEE International Conference on Acoustics, Speech, and Signal Processing, ICASSP 2010 - Proceedings.

[10] Le Montagner, Yoann, Marim Marcio.etc. Numerical evaluation of sampling bounds for nearoptimal reconstruction in compressed sensing[J].Proceedings - International Conference on Image Processing, ICIP, p 3073-3076, 2011, ICIP 2011: 2011 18th IEEE International Conference on Image Processing.

[11] Mun, Sungkwang. Residual reconstruction for block-based compressed sensing of video[J].Data Compression Conference Proceedings, p 183-192, 2011, Proceedings - DCC 2011: 2011 Data Compression Conference. 